\title{
Air Fraction Correction Optimisation in PET Imaging of Lung Disease
}

\author{
Francesca Leek, Andrew P. Robinson, Robert M. Moss, Frederick J. Wilson, Brian F. Hutton, Senior Member, IEEE \\ and Kris Thielemans, Senior Member, IEEE
}

\begin{abstract}
Accurate quantification of radiopharmaceutical uptake from lung PET/CT is challenging due to large variations in fractions of tissue, air, blood and water. Air fraction correction (AFC) uses voxel-wise air fractions, which can be determined from the CT acquired for attenuation correction (AC). However, resolution effects can cause artefacts in either of these corrections. In this work, we hypothesise that the resolution of the CT image used for AC should match that of the intrinsic resolution of the PET scanner but should approximate the reconstructed PET image resolution for AFC. Simulations and reconstructions were performed with the Synergistic Image Reconstruction Framework (SIRF) using phantoms with inhomogeneous attenuation (mu) maps, mimicking the densities observed in lung pathologies. Poisson noise was added to the projection data prior to OSEM reconstruction. AC was performed with a smoothed mu-map, the full-width-half-maximum (FWHM) of the 3D Gaussian kernel was varied $(0-10 \mathrm{~mm})$. Post-filters were applied to the reconstructed AC images (FWHM: 0 $-8 \mathrm{~mm}$ ). The simulated mu-map was independently convolved with another set of 3D Gaussian kernels, of varying FWHM (0 - $12 \mathrm{~mm})$, for AFC. The coefficient of variation $(\mathrm{CV})$ in the lung region, designed to be homogeneous post-AFC with optimised kernels, and the mean AFCstandardized uptake value (AFC-SUV) in the regions of simulated pathologies were determined. The spatial resolution of each postfiltered image was determined via a point-source insertion-andsubtraction method on noiseless data. Results showed that the $\mathrm{CV}$ was minimised when the kernel applied to the mu-map for AC matched that for the simulated PET scanner and the kernel applied to the mu-map for AFC matched the spatial resolution of the reconstructed PET image. This was observed for all postreconstruction filters and supports the hypothesis. Initial results from Monte Carlo simulations validate these findings.
\end{abstract}

\section{INTRODUCTION}

$\mathrm{T}$ ISSUE density variations contribute to the degradation of accuracy of quantitative PET/CT in the lung. The finite size of an imaging voxel, being significantly larger than the average alveolus size, results in a single voxel containing air and multiple tissue elements. This component of the partial volume effect is known as the tissue fraction effect (TFE); correcting for this has been shown to alter image interpretation in patients with idiopathic pulmonary fibrosis (IPF) [1][2].

Manuscript received December 13, 2020. This work was supported by an EPSRC Industrial CASE award (EP/T517628/1), the UK National Physical Laboratory through the National Measurement System Programmes Unit of the UK's Department of Business, Energy and Industrial Strategy, GlaxoSmithKline (BIDS3000035300) and UCL EPSRC Centre for Doctoral Training in Intelligent, Integrated Imaging in Healthcare (i4health).

F. Leek is with the Institute of Nuclear Medicine, University College London, London NW1 2BU, UK and the National Physical Laboratory, Teddington, Middlesex, W11 0LW, UK (email: francesca.leek.09@ucl.ac.uk).
Air fraction correction (AFC) assumes a simple model of the lung where the observed activity concentration is considered to be the result of the radiotracer distributed throughout the cellular component of the tissue (a combination of both parenchyma and blood) and a gas component, containing no activity [1]. The CT acquired for attenuation correction (AC) is utilised to determine voxel-wise fractions of unit volume occupied by non-aerated tissue. These air fractions (AF) can be used to account for the variable air content in the lung, providing an estimate of tracer uptake per gram of tissue.

The CT image is used for both $\mathrm{AC}$ and $\mathrm{AFC}$; however, the difference in resolution between PET and CT can cause artefacts in either of these corrections. We hypothesise that to minimise image artefacts caused by resolution effects, the CT resolution should match that of the PET scanner for AC but approximate the reconstructed PET image resolution for AFC. In this work, we test this hypothesis using analytic simulations of simple phantoms.

\section{METHODS}

The workflow for AFC of PET images, and the method by which this hypothesis was tested is shown in Fig. 1. Emission test-objects paired with inhomogeneous attenuation ( $\mathrm{mu}$ ) maps, that mimic the densities observed in lung pathologies, were simulated and reconstructed with a GE Discovery 710 template ( $\operatorname{span}=2$, maximum ring difference $=23$ ) using Software for Tomographic Image Reconstruction (STIR) [3] via the Synergistic Image Reconstruction Framework (SIRF) [4].

For determination of the optimal isotropic Gaussian kernel to be applied to the mu-map for $\mathrm{AC}, h_{A C}(x)$, a $150 \mathrm{~mm} \emptyset \times 78 \mathrm{~mm}$ cylindrical test-object was used, test-object 1 , as can be seen in Fig. 2. The mu-map included two spherical inserts $(22 \mathrm{~mm} \emptyset$, $0.0406 \mathrm{~cm}^{-1} ; 13 \mathrm{~mm} \emptyset, 0.0031 \mathrm{~cm}^{-1}$ ) positioned on the central axial slice and a soft tissue / healthy lung boundary $(0.0960$ / $0.0191 \mathrm{~cm}^{-1}$ ). Test-object 2, used in the determination of the optimal isotropic Gaussian kernel to be applied to the mu-map for $\mathrm{AFC}, h_{A F C}(x)$, is depicted in Fig. 1 . The $300 \times 200 \mathrm{~mm} \emptyset \mathrm{x}$

B. F. Hutton and K. Thielemans are with the Institute of Nuclear Medicine, University College London.

A. P. Robinson is with National Physical Laboratory, Christie Medical Physics and Engineering, The Christie NHS Foundation Trust, UK and Schuster Laboratory, School of Physics and Astronomy, the University of Manchester, UK.

R. M. Moss is with the Department of Medical Physics and Biomedical Engineering, University College London, Gower Street, London WC1E 6BT, UK.

F. J. Wilson is with GlaxoSmithKline Research \& Development Limited Gunnels Wood Road, Stevenage, Herts, SG1 2NY, UK. 
$78 \mathrm{~mm}$ elliptical cylinder $\left(0.0960 \mathrm{~cm}^{-1}\right)$ contained two $130 \mathrm{x}$ $120 \mathrm{~mm} \varnothing$ lungs $\left(0.0191 \mathrm{~cm}^{-1}\right)$; the simulated emission was designed to produce a homogeneous $\mathrm{AFC}-\mathrm{SUV}=3$ in the lungs (Fig. 1(f)). The mu-map contained a 2D high-resolution CT patch of centrilobular emphysema (CLE; 31 x $31 \mathrm{~mm}, 0.0031-$ $0.0586 \mathrm{~cm}^{-1}$ ) [5], repeated on the three central slices of the left lung; the right lung contained a spherical insert representative of IPF density $\left(22 \mathrm{~mm} \emptyset ; 0.0406 \mathrm{~cm}^{-1}\right)$.

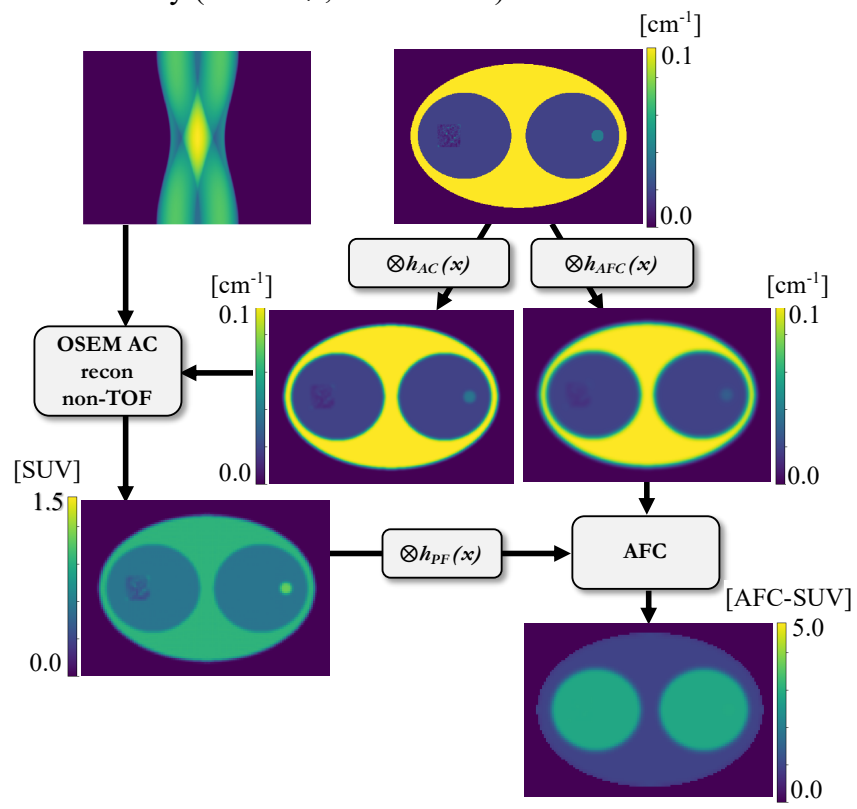

Fig. 1. AFC workflow depicting test-object 2, noise-less scenario: (a) simulated emission, smoothed to $4.7 \mathrm{~mm}$ FWHM prior to forward-projection; (b) simulated inhomogeneous mu-map; (c) mu-map $\otimes h_{A C}(x)$ for AC; (d) AC reconstructed PET; (e) mu-map $\otimes h_{A F C}(x)$ for AFC; (f) AC-AFC PET: AC PET $\otimes h_{P F}(x)$ and divided by $k_{v}(1 / \mathrm{AF})$ voxel-wise; $k_{v}$ determined from (1).

The simulated emission data and mu-map (voxel size: $0.78 \mathrm{x}$ $0.78 \times 3.27 \mathrm{~mm}$ ) were convolved with an isotropic Gaussian kernel with a full-width-half-maximum (FWHM) of $4.7 \mathrm{~mm}$ prior to forward-projection, to approximate the intrinsic spatial resolution of a PET scanner [6].

OSEM reconstruction with 2000 iterative updates and 6 subsets (one update uses one subset of data), was performed; the FWHM of $h_{A C}(x)$ was varied from $0-10 \mathrm{~mm}$ in $1 \mathrm{~mm}$ increments. The reconstructed voxel-size was $2.71 \times 2.71 \times 3.27$ $\mathrm{mm}$. Time of flight (TOF) was not enabled.

An isotropic Gaussian post-filter, $h_{P F}(x)$, of varying FWHM (0-8 mm, $2 \mathrm{~mm}$ increments), was applied to each of the AC reconstructed PET images, to mimic clinical practice while also allowing the study of different resolutions in the reconstructed image.

The spatial resolution of each smoothed attenuationcorrected PET image was determined on the central axial slice of noiseless data, via a point-source insertion-and-subtraction method. A one voxel point-source was positioned at multiple points in the field-of-view (FOV) with various densities and structure; it was ensured that the reconstructed point-source contrast was less than 0.1 [7].
$h_{A F C}(x)$, of varying FWHM (0-12 mm, $1 \mathrm{~mm}$ increments) was independently applied to the mu-map for voxel-wise AF determination and subsequent AFC.

Given the linear scaling between Hounsfield Units (HU) and linear attenuation coefficients in the lung [8] the relationship between the fraction of tissue in each voxel, $k_{v}$, and lung density, as defined by Lambrou et al. [1] can be expressed as:

$$
\mu_{v}=k_{v} \mu_{\text {tissue }}+\left(1-k_{v}\right) \mu_{\text {air }}
$$

where $\mu_{v}, \mu_{\text {tissue }}$ and $\mu_{\text {air }}$ are linear attenuation coefficients for $511 \mathrm{keV}$ photons in the mu-map voxel, soft tissue and air, respectively. The reconstructed PET count distribution was divided by $k_{v}$ on a voxel-wise basis so as to represent uptake per gram of tissue rather than per unit volume.

The optimal kernels for $\mathrm{AC}$ and $\mathrm{AFC}$ were determined independently. In both cases, Poisson noise was added to the uniform emission projection data to simulate a non-TOF clinical dataset (number of prompts $\approx 1 \mathrm{e}^{8}$ ).

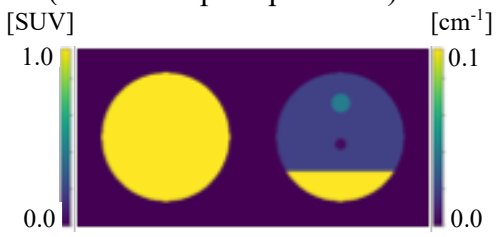

(a)

(b)

Fig. 2. Test-object 1: (a) simulated homogeneous emission; (b) simulated inhomogeneous mu-map.

The coefficient of variation (CV) in the central slices of the test-object, for $h_{A C}(x)$ determination, and the 'lungs' for $h_{A F C}(x)$ determination, was calculated on the mean image of 100 noise realisations for each test-object. Test-objects were designed to produce uniform activity distributions post-AC (test-object 1) and post-AC-AFC (test-object 2), when kernels were optimised. To remove edge-of-phantom artefacts from the analysis, the volume of interest (VOI) was eroded in both the transaxial and axial directions. The mean AFC-standardized uptake value (AFC-SUV) in the CLE and IPF regions were also determined for each triple of blurring kernels.

\section{RESULTS}

Image artefacts were minimised $(\mathrm{CV}=3.21 \%)$ in the non$\mathrm{AFC}$ reconstructed images of test-object 1 when the mu-map resolution for $\mathrm{AC}$ matched that of the simulated PET scanner, Fig. 3. When PET scanner and mu-map resolutions were mismatched artefacts were seen at tissue density boundaries. When the mu-map resolution was higher than the PET scanner resolution, counts on the low-density side of the tissue boundaries were artificially decreased. The converse was true for a lower mu-map resolution than the PET scanner resolution. This agrees with findings for FBP reconstruction [9]. 


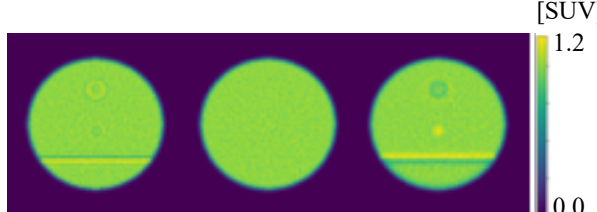

(a)

(b)

(c)

Fig. 3. OSEM reconstruction (2000 updates, 6 subsets, mean of 100 noise realisations) of test-object 1 with various $h_{A C}(x)$; (a) $0.0 \mathrm{~mm}$; (b) $4.7 \mathrm{~mm}$; (c) $10.0 \mathrm{~mm}$. Simulated PET scanner resolution $=4.7 \mathrm{~mm}$.

The mu-map resolution for $\mathrm{AC}$ was therefore matched to the PET scanner resolution for determination of the optimal kernel for AFC. Fig. 4 depicts the artefacts produced in test-object 2 at tissue density boundaries in the AFC reconstructed images when there was a mismatch in the reconstructed image resolution and the mu-map used in the determination of the AFs. These artefacts were reduced $(\mathrm{CV}=3.20 \%)$ when $h_{A F C}(x)$ approximated the measured image resolution, which in Fig. 4 was $7.4 \mathrm{~mm}$.

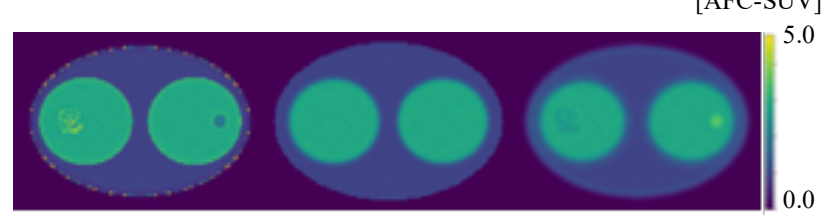

(a)

(b)

(c)

Fig 4 AC-AFC images of test-object 2 (OSEM reconstruction: 2000 updates, 6 subsets), mean of 100 noise realisations. AFs determined from mumap $\otimes h_{A F C}(x)$ with various FWHM, $h_{A C}(x)=4.7 \mathrm{~mm}, h_{P F}(x)=6.0 \mathrm{~mm}$; (a) 0.0 $\mathrm{mm}$; (b) $7.0 \mathrm{~mm}$; (c) $12.0 \mathrm{~mm}$. The mean measured image resolution was 7.4 $\mathrm{mm}$.

The change in mean AFC-SUV in the CLE and IPF features as $h_{A F C}(x)$ is varied is shown in Fig. 5. It can be seen that the optimal smoothing for AFC-SUV accuracy, for both the highand low-density lung pathologies, approximates the measured image resolution. This was observed for all post-filters.

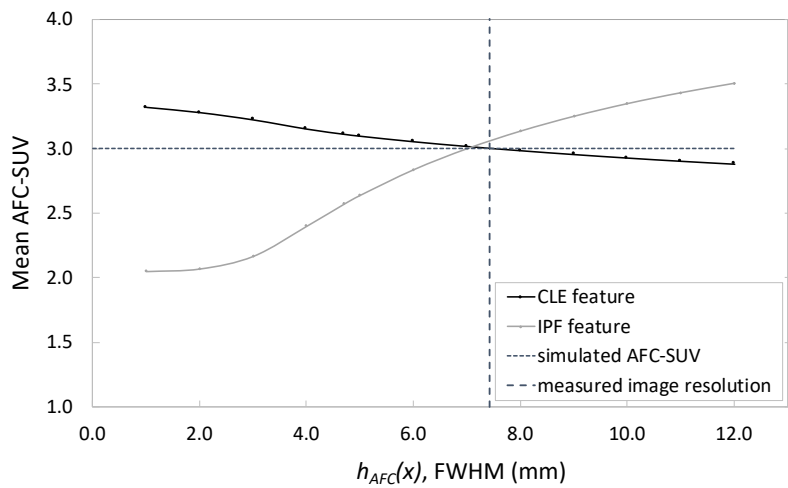

Fig. 5. Mean AFC-SUV in CLE and IPF features for various $h_{A F C}(x)$, $h_{A C}(x)=4.7 \mathrm{~mm}, h_{P F}(x)=6.0 \mathrm{~mm}$, OSEM reconstruction ( 2000 updates, 6 subsets). Dashed vertical line indicates the measured image resolution; simulated AFCSUV represented by the horizonal dotted line.

It can be seen that when the mu-map resolution is higher than the reconstructed PET resolution, AFC-SUV in the low-density CLE feature is artificially increased $(10.6 \%$ at sampling resolution) but decreased $\left(-4.0 \%\right.$ for $\left.h_{A F C}(x)=12 \mathrm{~mm}\right)$ when the mu-map resolution is lower than the PET image resolution. The converse is true for higher density IPF region $(-31.7 \%$ at sampling resolution; $16.7 \%$ for $\left.h_{A F C}(x)=12 \mathrm{~mm}\right)$.
Large numbers of iterative updates were needed to achieve measured image resolution stability and minimise image artefacts. Fig. 6 shows the measured transaxial image resolution in the lung within different densities and structure.

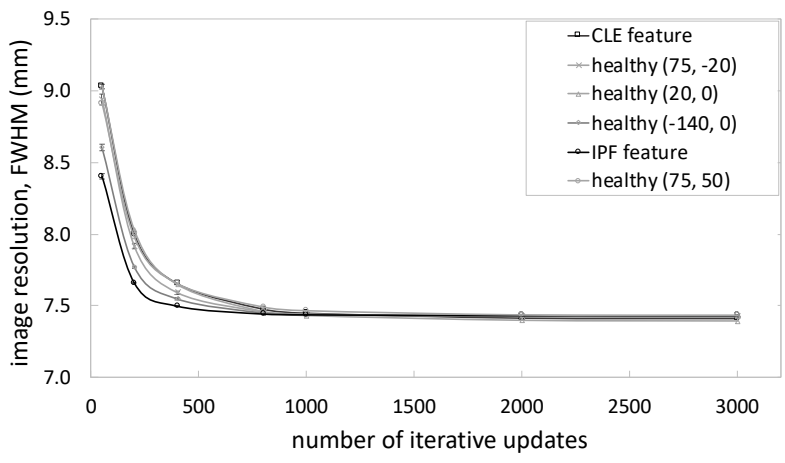

Fig. 6. Measured transaxial image resolution for noiseless point sources at various positions in the lung (healthy (radial offset CFOV (mm), tangential), CLE and IPF) for increasing numbers of non-TOF iterative updates, 6 subsets. $h_{A C}(x)=4.7 \mathrm{~mm}, h_{A F C}(x)=7.0 \mathrm{~mm}, h_{P F}(x)=6.0 \mathrm{~mm}$; error bars depict one standard deviation.

Fig. 7 depicts the image artefacts evident in the underconverged images. Improvement in VOI homogeneity becomes less apparent beyond 1000 iterative updates.

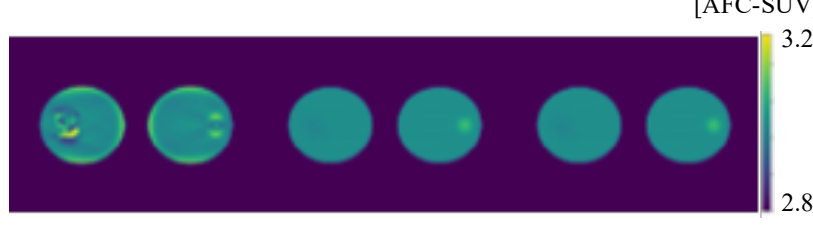

(a)

(b)

Fig. 7. Central slice of noiseless AC-AFC PET $h_{A C}(x)=4.7 \mathrm{~mm}$ $h_{A F C}(x)=7.0 \mathrm{~mm}, h_{P F}(x)=6.0 \mathrm{~mm}$ (a) 50 updates; (b) 1000 updates; (c) 2000 updates; 6 subsets.

\section{DiscuSSION AND CONCLUSIONS}

Artefacts observed in the AC-AFC PET images at tissue density boundaries were minimised when the $\mathrm{CT}$ resolution matched the simulated PET scanner resolution for $\mathrm{AC}$ and approximated the PET image resolution for AFC. This supports the initial hypothesis. Residual artefacts were likely due to spatially variant image resolution; in the current work, only spatially invariant kernels at selected isotropic $h_{A C}(x)$ and $h_{A F C}(x)$ were investigated for each $h_{P F}(x)$.

A mismatched mu-map resolution has opposing effects on the resultant activity density for AC and AFC. It has been demonstrated that, in non-TOF PET, the effects of attenuation mismatch are local [10]. Therefore, if the same mu-map resolution is used for both $\mathrm{AC}$ and $\mathrm{AFC}$, the effect of the mismatch for AC can be partially corrected for by the AFC. The effect of TOF on these artefacts is to be investigated.

Large numbers of iterative updates were needed to achieve stability in the measured image resolution. This has implications when designing a clinical lung imaging protocol that includes AFC.

Initial results from Monte Carlo simulations (GATE v8.2 [11]) validate these findings. The effect of resolution mismatches on fine scale structures is being investigated. 


\section{REFERENCES}

[1] T. Lambrou, A.M. Groves, K. Erlandsson, N. Screaton, R. Endozo, T. Win, J.C. Porter and B.F. Hutton, "The importance of correction for tissue fraction effects in lung PET: preliminary fndings", Eur J Nucl Med Mol Imaging, 38:2238-2246, 2011

[2] T. Win, B.A. Thomas, T. Lambrou, B.F. Hutton, N.J. Screaton, J.C. Porter, T.M. Maher, R. Endozo, R.I. Shortman, A. Afaq, P. Lukey, P.J. Ell and A.M. Groves, "Areas of normal pulmonary parenchyma on HRCT exhibit increased FDG PET signal in IPF patients", Eur J Nucl Med Mol Imaging, 41:337-342, 2014

[3] K. Thielemans, C. Tsoumpas, S. Mustafovic, T. Beisel, P. Aguiar, N. Dikaios and M.W. Jacobson, "STIR: software for tomographic image reconstruction release 2", Phys Med Biol 57(4):867-883, 2012.

[4] E. Ovtchinnikov et al. "SIRF: Synergistic Image Reconstruction Framework", Computer Phys Comms, 249:107087, 2020.

[5] L. Sørensen, S.B. Shaker and M. de Bruijne, "Quantitative Analysis of Pulmonary Emphysema Using Local Binary Patterns", IEEE Trans. Med. Imaging 29(2):559-569, 2010.

[6] V. Bettinardi, L. Presotto, E. Rapisarda, M. Picchio, L. Gianolli, M.C. Gilardi, "Physical Performance of the new hybrid PET/CT Discovery690", Clin Transl Imaging 2:199-218, 2014.

[7] K. Gong, S.R. Cherry and Jinyi Qi, "On the assessment of spatial resolution of PET sustems with iterative image reconstruction", Phys Med Biol, vol. 61, no. 5, pp. 193-202, 2016.

[8] C. Burger, G. Goerres, S. Schoenes, A. Buck, A.H.R. Lonn and G.K. von Schulthess, "PET attenuation coefficients from CT images: experimental evaluation of the transformation of CT into PET 511-keV attenuation coefficients", Eur J Nucl Med 29:922-927, 2002

[9] S.R. Meikle, M. Dahlbom and S.R. Cherry, "Attenuation Correction Using Count-Limited Transmission Data in Positron Emission Tomography", J. Nucl. Med. 34:143-144, 1993.

[10] K. Thielemans, E. Asma, R.M. Manjeshwar, A. Ganin, T.J. Spinks, "Image-based correction for mismatched attenuation in PET images", IEEE NSS CR 2008, 5292-5296.

[11] S. Jan, G. Santin, D. Strul, S. Staelens, K. Assié, D. Autret, S. Avner, R. Barbier, M. Bardiès and P.M. Bloomfield, "GATE: a simulation toolkit for PET and SPECT”, Phys Med Biol 49(19):4543-4561, 2004. 